\title{
ALGEBRA FOR HECKOID GROUPS
}

\author{
ROBERT RILEY
}

\begin{abstract}
We introduce an infinite collection of (Laurent) polynomials associated with a 2-bridge knot or link normal form $K=(\alpha, \beta)$. Experimental evidence suggests that these "Heckoid polynomials" define the affine representation variety of certain groups, the Heckoid groups, for $K$. We discuss relations which hold in the image of the generic representation for each polynomial. We show that, with a certain change of variable, each Heckoid polynomial divides the nonabelian representation polynomial of $L$, where $L$ belongs to an infinite collection of 2-bridge knots/links determined by $K$ and the Heckoid polynomial. Finally, we introduce a "precusp polynomial" for each 2-bridge knot normal form, and show it is the product of two (possibly reducible) nonconstant polynomials. We are preparing a sequel on the Heckoid groups and the evidence for some of the geometrical assertions stated in the introduction.
\end{abstract}

A Heckoid group $\Gamma \subset \mathrm{SL}_{2}(\mathbb{C})$ is a Kleinian group (of second kind, acting discontinuously on an open set of the extended complex plane) which contains elliptics and is generated by parabolics in a particular way: there is a parabolic $P \in \Gamma$ and an elliptic $S \in \mathrm{SL}_{2}(\mathbb{C})$ such that $\Gamma=\left\langle S^{\nu} P S^{-\nu}: \nu \in \mathbb{Z}\right\rangle$. All known examples of such groups are geometrically finite, but we shall also require geometric finiteness just to be on the safe side. The classical Hecke groups are essentially the first examples of Heckoid groups, the caveat being that $E$. Hecke took $\langle P, S\rangle$ as his group, whereas for us $S$ only sometimes belongs to its Heckoid group. The "modest example" of [6] is an even Heckoid group for the pretzel knot $(3,3,3)$, and in [1] M. Grayson showed that it gives a hyperbolic orbifold structure of infinite volume to the knot complement $S^{3}-(3,3,3)$. This sort of thing is what the present paper is leading up to, but is not actually about.

We shall mainly be studying (Laurent) polynomials of various sorts associated with nonabelian representations in $\mathrm{SL}_{2}(\mathbb{C})$ of a 2-bridge knot or link group, $\pi K$. This group is a 2-generator one relator group, $\pi K=\mid x_{1}, x_{2}:$ rel $\mid$, and as preparation for representing it we substitute matrices $C, D$ for $x_{1}, x_{2}$ in rel, where $C, D \in \mathrm{SL}_{2}(R)$ are in normal form over a suitable (Laurent) polynomial algebra $R$ over $\mathbb{Z}$. Then $\operatorname{rel}(C, D)=E$ is equivalent to four polynomial equations in $R$, but in our 2-bridge case only one is needed, say $\Phi_{K}=0$. In [7] we studied the polynomial $\Phi_{K}$ for a 2-bridge knot and determined many of its properties. In $\S 1$ below we review this material and add one new property that we found too late for [7]. However the primary purpose of $\S 1$ is to switch from the nonunimodular normal form $C, D$ used in [7] to a unimodular normal

Received by the editors September 1, 1990.

1991 Mathematics Subject Classification. Primary 20H99; Secondary 57M25. 
form for the rest of this paper. A simple change of variable will convert the statements of the results here back to the version of [7], with the advantage of reducing the degrees of the polynomials, but a unimodular normal form is best for the proofs. In $\S 2$ we give the corresponding account for 2-bridge links. This material has not previously been published, but it is not new. Our colleague Craig Squier obtained a nonabelian representation polynomial for a 2bridge link by the same method, although with "Fricke trace arguments" rather than normal forms. (Of course this is closely related to a general theorem of W. Thurston [9]. In our language, Thurston obtained the factor of $\Phi_{K}$ for the hyperbolic structure on the complement $S^{3}-K$.) In $\S 3$ we work out some matrix and polynomial identities for later use. The Heckoid results are expressed in terms of the Morgan-Voyce polynomials $f_{n}(x), g_{n}(x) \in \mathbb{Z}[x]$, defined for $n \in \mathbb{Z}$ by

$$
\left[\begin{array}{cc}
1+x & 1 \\
x & 1
\end{array}\right]^{n}=\left[\begin{array}{cc}
f_{n}(x) & g_{n-1}(x) \\
x g_{n-1}(x) & f_{n-1}(x)
\end{array}\right] .
$$

In $\S 4$ we reach the Heckoid algebra. For a 2-bridge normal form $K$ we define a variable $v_{K} \in R$, where $R$ is the polynomial ring for $C, D$. When $K$ is a knot, $v_{K}=u \Phi_{K}^{2}$, and when $K$ is a link, $v_{K}$ is the slightly more complicated expression (4.2).

Theorem A. There are words $T, V$ in $\langle C, D\rangle$ determined by $K$ ( $V$ is essentially $\operatorname{rel}(C, D)$ and $T$ is the first half of $V)$ with the following properties. The statement involves an integral parameter $q \geq 3$ and depends on the parity of $q$.

(a) $q=2 n+1$ is odd: Let $U=V^{n} T$. Then in $\operatorname{SL}_{2}\left(R /\left(f_{n}\left(v_{K}\right)\right)\right)$ one has $U^{2}=V^{q}=-E, U C U^{-1}=D^{-1}$ if $K$ is a knot, and $U C U^{-1}=C^{-1}$, $U D U^{-1}=D^{-1}$ if $K$ is a link.

(b) $q=2 n+2$ is even: In $\mathrm{SL}_{2}\left(R /\left(g_{n}\left(v_{K}\right)\right)\right)$ one has $V^{q}=E$.

The main result of $\S 4$ is a more precise version of Theorem $A$. The Heckoid group of index $q$ for $K$ is the group $\langle C, D\rangle$ where the right complex numbers are substituted for the variables of $R$. The requirements are that $f_{n}\left(v_{K}\right)=0$ or $g_{n}\left(v_{K}\right)=0$, that $C$ and $D$ be parabolic, and that $\langle C, D\rangle$ be discrete in $\mathrm{SL}_{2}(\mathbb{C})$. This last requires that we use the correct choice from a set of conjugate algebraic integers for one parameter.

Our next main topic is the factorization of the nonabelian representation polynomial $\Phi_{K}$ of a 2-bridge normal form $K$. In this introduction we restrict the discussion to knots, although the results are extended to links in $\S \S 5$ and 6. We know of three cases where $\Phi_{K}(t, u) \in R=\mathbb{Z}\left[t, t^{-1}, u\right]$ is reducible for the 2-bridge knot normal form $(\alpha, \beta)$.

Red I. $\beta^{2} \equiv+1(\bmod 2 \alpha)$ but $\beta \not \equiv \pm 1(\bmod 2 \alpha)$. Knots of this type have an alternating diagram ("plumbing representation") corresponding to a palindromic integral sequence $a_{1}, a_{2}, \ldots, a_{r}$, and admit a symmetry which inverts the order of the $a_{j}$. By considering the action of this symmetry on the collection of ideal points of the representation space for the knot, T. Ohtsuki showed in [3] that $\Phi_{K}$ for $K$ in Red I is always reducible. Our calculations using the symbolic manipulation system MAPLE suggest that Red I can be decomposed into an infinite collection of doubly infinite sequences so that for the knots $K(n)$ of one sequence, $\Phi_{K(n)}=F_{n} \cdot G_{n}$, where $F_{n}, G_{n}$ belong to $\mathbb{Z}\left[t, t^{-1}, u\right]$ and 
have explicit expressions as forms on Morgan-Voyce polynomials $f_{m}(\xi), g_{m}(\xi)$. Here $m=|n|-1$ and $\xi \in \mathbb{Z}\left[t, t^{-1}, u\right]$. We expect further that the excellent factor $F_{n}$ (corresponding to the hyperbolic structure) is only a linear form on $f_{m}(\xi), g_{m}(\xi)$, whence the strange factor $G_{n}$ will be a cumbersome form of high degree. Our experience suggests that when a knot admits an unusually large group of symmetries which preserve both space and knot orientation then the affine variety of nonabelian representations of the knot group has extra algebraic components. Red I is part of the evidence for this.

Red II. For each 2-bridge knot $K$ and for each odd integer $q$ there is an infinite collection, call it $K(q)$, of 2-bridge knots $K^{*}$ with the property that each knot group $\pi K^{*}$ has a surjective homomorphism on $\pi K$ which sends meridian to meridian and longitude to (longitude) ${ }^{q}$. (This incorporates a suggestion of Tomotada Ohtsuki who improved our construction of $K(q)$.) Obviously $\Phi_{K} \mid \Phi_{K^{*}}$ for all $K^{*} \in K(q)$.

Red III. Theorem B. Let $K=(\alpha, \beta)$ be a 2-bridge knot normal form. For integers $r \geq 2, q=2 n+1 \geq 3$, and $e= \pm 1$, let $K^{*}$ be the 2-bridge knot normal form $\left(\alpha^{r} q, \alpha^{r-1} q(\alpha-\beta)+e\right)$. Then

$$
f_{n}\left(v_{K}(t, \tilde{u})\right) \mid \Phi_{K^{*}}(t, u) \text {, where } \tilde{u}=\left(t^{-1}-t\right)^{2}-u \text {. }
$$

Note that this factor is the representation polynomial for the $q$ th Heckoid group of $K$, with a change of variable. Therefore $\pi K^{*}$ will have a parabolic representation on this odd Heckoid group. The even Heckoid groups of $K$ are also the images of parabolic representations of certain 2-bridge link groups. We prove Theorem $B$ and its analogues for links in $\S \S 5$ and 6.

Our last main topic is a factorization of the "precusp polynomial" $4+$ $u \Phi_{K}^{2}(1, u)$ of a 2-bridge knot normal form $K=(\alpha, \beta)$.

Theorem C. The polynomial $4+u \Phi_{K}^{2}(1, u)$ has a factor $\Lambda^{*}(-u) \in \mathbb{Z}[u]$ of the shape $1+c_{1} u+\cdots+c_{m-1} u^{m-1}+u^{m}, m=(\alpha-1) / 2$.

We identify this factor and prove the theorem in $\S 7$. But as our proof gives no clue as to what this is really about we outline the context of Theorem $C$ here. Consider the collection of groups

$$
\begin{gathered}
\Gamma\{\omega\}=\langle A, B\{\omega\}\rangle, \quad A=\left[\begin{array}{ll}
1 & 1 \\
0 & 1
\end{array}\right]=C\{1\}, \\
B\{\omega\}=\left[\begin{array}{cc}
1 & 0 \\
-\omega & 1
\end{array}\right]=D\{1, \omega\},
\end{gathered}
$$

where $\omega \in \mathbb{C}$. Let

$$
\begin{aligned}
& \mathscr{R}=\text { interior(closure }\{\omega \in \mathbb{C}: \Gamma\{\omega\} \text { is not free of rank } 2\}), \\
& \mathscr{D}=\{\omega \in \mathscr{R}: \Gamma\{\omega\} \text { is discrete }\} .
\end{aligned}
$$

In 1978-1979 we produced a computer-drawn diagram of the portion of $\mathscr{R}$ in the first quadrant of $\mathbb{C}$ which displayed the influences of $\mathscr{D}$ on the shape of $\mathscr{R}$. The calculations for the diagram were pushed far enough to suggest that all essential phenomena were depicted, and if the diagram does not lie the following assertions are true. First, each $\omega \in \mathscr{D}$ such that $\Gamma\{\omega\}$ is torsion-free 
corresponds to the hyperbolic structure on a 2-bridge knot or link complement. (There is a mild exception for $\omega=0$, which corresponds to the Hopf link $(2,1)$.) Each $\omega \in \mathscr{D}$ such that $\Gamma\{\omega\}$ is not torsion-free has $\Gamma\{\omega\}$ as Heckoid group of some 2-bridge knot or link, or of the trivial knot $O$. The points $\omega_{q}(K), q=3,4, \ldots$, determining the Heckoid groups for a 2-bridge normal form (or $O)$ converge to a cusp $\omega_{\infty}(K)$ of bdry $\mathscr{R}$. The group $\Gamma\left\{\omega_{\infty}(K)\right\}$ is a subgroup of the image of an excellent parabolic representation of the group of some 2- or 3-component link $L$ found by Thurston in a different context. If $K$ is a knot, $K \neq O$, then $\omega_{\infty}(K)$ is a root of the cofactor of $\Lambda^{*}(-u)$ in the precusp polynomial for $K$. So Theorem $C$ is really asserting that the cusps of bdry $\mathscr{R}$ for 2-bridge knots are algebraic integers of degree $(1+\operatorname{det} K) / 2$ at most, instead of $\operatorname{det} K$ itself. Hence Theorem $C$ is an aid to the explicit calculation of these cusps.

We add a postscript, $\S 8$, which briefly describes the current status of the Heckoid algebra for groups with more than two generators.

Two of the background results below, Proposition 1 of $\S 1$ and Lemma 1 of $\S 2$, were proved at Binghamton when we were partially supported by an NSF grant. All the rest (except examples and parts of $\S 3$ ) were obtained during the first five months of 1990 while the author was on sabbatical leave at Southampton University. Nevertheless the theorems of $\S 4$ are direct descendents of the results of Chapter 8 of our $1979 \mathrm{Ph} . D$. thesis, written at Southampton University under the supervision of Dr. David Singerman, when the author was supported by the Science Research Council of the U.K. (1976-1979). Actually, we had found instances of Theorem B for parabolic representations in 1971 and of Theorem C for torus knots $(\alpha, 1)$ by 1975 , but we are still not sure that we have found the proper contexts for the results.

This paper is a direct continuation of [7], which continues [4], and any unexplained notation will be found in those papers.

\section{REPRESENTATIONS OF TWO-BRIDGE KNOTS}

Let $\alpha$ be an odd integer $\geq 3$. A 2-bridge kmot group $\pi K$ of determinant $\alpha$ is a group presented by

$$
\pi K=\left|x_{1}, x_{2}: w x_{1}=x_{2} w\right|,
$$

where

$$
w=x_{1}^{\varepsilon_{1}} x_{2}^{\varepsilon_{2}} x_{1}^{\varepsilon_{3}} \cdots x_{2}^{\varepsilon_{\alpha-1}}
$$

in which

$$
\varepsilon_{\nu}=\varepsilon_{\alpha-\nu}= \pm 1 \quad \text { for } \nu=1, \ldots, \alpha-1 .
$$

Such a kmot group is a knot group when $\vec{\varepsilon}=\left(\varepsilon_{1}, \ldots, \varepsilon_{\alpha-1}\right)$, the exponent sequence, is determined by a second parameter $\beta$, which is an odd integer relatively prime to $\alpha$, by the rule

$$
\varepsilon_{\nu}=\operatorname{sign}(\operatorname{mods}(\nu \beta, 2 \alpha)),
$$

where $\operatorname{mods}(c, d)$ is the unique integer $e \equiv c(\bmod d)$ with $-d / 2<e \leq d / 2$. This rule not only implies the palindromic symmetry (1.1c) which all our work depends on, but also implies by a geometric argument that if

$$
\bar{w}=x_{1}^{-\varepsilon_{1}} x_{2}^{-\varepsilon_{2}} x_{1}^{-\varepsilon_{3}} \cdots x_{2}^{-\varepsilon_{\alpha-1}}
$$


then $\bar{w} x_{1}=x_{2} \bar{w}$ is a consequence of $w x_{1}=x_{2} w$ in $\pi K$. Since kmots do not exist as geometric objects there is no analogous argument for proper kmot groups (those whose presentation (1.1) does not coincide with a 2-bridge knot presentation), and indeed R. Lyndon (letter to the author) showed that $\bar{w} x_{1}=x_{2} \bar{w}$ does not hold in the kmot group corresponding to $\vec{\varepsilon}=(1,-1,-1,-1,-1,1)$. In our previous papers on 2-bridge knots we may have interchanged the roles of $w, \bar{w}$, but this has no effect on the polynomials we study.

The trivial knot $O$, whose group $\pi O$ is isomorphic to $|x: \varnothing|$, can be presented by (1.1) using $\alpha=1$ and $w=E$ (the identity). We usually do not include $O$ among the 2-bridge knots, but including $O$ will be natural when we get to the Heckoid groups.

In [7] we based the study of the curve of nonabelian representations of a 2-bridge kmot group on the normal form

$$
C=\left[\begin{array}{ll}
t & 1 \\
0 & 1
\end{array}\right], \quad D=\left[\begin{array}{cc}
t & 0 \\
-t u & 1
\end{array}\right]
$$

So

$$
W=w(C, D)=C^{\varepsilon_{1}} D^{\varepsilon_{2}} C^{\varepsilon_{3}} \cdots D^{\varepsilon_{\alpha-1}}=\left[\begin{array}{ll}
w_{11} & w_{12} \\
w_{21} & w_{22}
\end{array}\right] \in M_{2}\left(\mathbb{Z}\left[t, t^{-1}, u\right]\right)
$$

gives rise to the nab-representation polynomial

$$
\begin{gathered}
\Phi_{\pi K}(t, u)=\Phi(t, u)=t^{\sigma / 2}\left[w_{11}+(1-t) w_{12}\right], \\
\sigma=\varepsilon_{1}+\varepsilon_{2}+\cdots+\varepsilon_{\alpha-1} .
\end{gathered}
$$

The equations on the variables $t, u$ expressing $W C=D W$ are $\Phi(t, u)=0$ and

$$
w_{21}+t u w_{12}=0
$$

but the palindrome $(1.1 \mathrm{c})$ makes $(1.8)$ a polynomial identity. The numerical coefficients of $\Phi(t, u)$ alternate in sign in a manner determined by Proposition 1 , a weaker result than what our calculated examples suggest is true.

Proposition 1. The nonzero numerical coefficients in $(-1)^{m} \Phi_{\pi K}(-t, u)$ are all positive, where $m=(\alpha-1) / 2$.

Proof. One observes that all numerical coefficients in entries of the four matrices $C\{-t\}^{\gamma} D\{-t, u\}^{\delta}$, where $\gamma$ and $\delta$ are \pm 1 , are nonnegative. The matrix $W(-t, u)$ is the product of $m$ such matrices, and so all its numerical coefficients are nonnegative. Hence the nonzero coefficients in

$$
\Phi(-t, u)=(-1)^{\sigma / 2} t^{\sigma / 2}\left[w_{11}(-t, u)+(1+t) w_{12}(-t, u)\right]
$$

all have one sign. But we showed in [7, $§ 2$, Lemma 2] that the coefficient of $u^{m}$ in $\Phi(t, u)$ is $(-1)^{m}$, whence the coefficient of $u^{m}$ in $(-1)^{m} \Phi(-t, u)$ is +1 , proving Proposition 1.

Unfortunately, as we confessed at the end of $[7, \S 1]$, the above normal form $C, D$ rather confuses us, because $\operatorname{det} C \equiv \operatorname{det} D \equiv t \not \equiv 1$, whereas the trace arguments that we have in mind work best when all determinants are $\equiv 1$. So henceforth we use instead the unimodular normal form

$$
C=C\{t\}=\left[\begin{array}{cc}
t & 1 \\
0 & t^{-1}
\end{array}\right], \quad D=D\{t, u\}=\left[\begin{array}{cc}
t & 0 \\
-u & t^{-1}
\end{array}\right]
$$


for the images of the standard meridian generators of our kmot group. But this changes the use of the variable $t:$ the variables $t, u$ of the normal form (1.4) and its consequences correspond to $t^{2}, u$ of (1.9). We conserve notation as much as possible, so we still write $W=C^{\varepsilon_{1}} D^{\varepsilon_{2}} \cdots D^{\varepsilon_{\alpha-1}}$, etc. The equations expressing $W C=D W$ are now

$$
\begin{gathered}
\Phi_{\pi K}(t, u)=\Phi(t, u)=w_{11}+\left(t^{-1}-t\right) w_{12}, \\
w_{21}+u w_{12}=0 .
\end{gathered}
$$

We have $\Phi(t, u)$ of (1.6) equals $\Phi\left(t^{2}, u\right)$ of (1.10), and so Proposition 1 asserts $(-1)^{m} \Phi(i t, u)$ has positive coefficients, where $i^{2}=-1$. We hope to avoid confusion by never referring to the nonunimodular normal form and its consequences again. The price of unimodularity is that our polynomials have degree in $t, t^{-1}$ twice the natural value, e.g., our Alexander polynomial $\Delta(t)=\Phi(t, 0)$ would commonly be written $\Delta\left(t^{2}\right)$. We do gain a further advantage though, since writing $\tau=t+t^{-1}=\operatorname{tr} C=\operatorname{tr} D$ allows us to write $\Psi_{\pi K}(\tau, u) \equiv \Phi_{\pi K}(t, u)$, where $\Psi(\tau, u) \in \mathbb{Z}[\tau, u]$ (cf. Proposition 1 of [7]). Here the variables $\tau, u$ are (essentially) traces, whence $\Psi$ is our most natural polynomial.

\section{REPRESENTATIONS OF 2-BRIDGE LINK GROUPS}

Let $\alpha$ be an even integer $\geq 2$. A 2-bridge limk group of determinant $\alpha$ is a group presented by

$$
\pi L=\left|x_{1}, x_{2}: w x_{1}=x_{1} w\right|,
$$

where

$$
w=x_{2}^{\varepsilon_{1}} x_{1}^{\varepsilon_{2}} x_{2}^{\varepsilon_{3}} \cdots x_{2}^{\varepsilon_{n-1}}
$$

in which

$$
\varepsilon_{\nu}=\varepsilon_{\alpha-\nu}= \pm 1 \text { for } \nu=1, \ldots, \alpha-1 .
$$

Such a limk group is a link group when the exponent sequence $\vec{\varepsilon}=\left(\varepsilon_{1}, \varepsilon_{2}, \ldots\right.$, $\left.\varepsilon_{\alpha-1}\right)$ is determined by a second parameter $\beta$, where $\beta$ is an odd integer relatively prime to $\alpha$, by the rule (1.2) again. A 2-bridge link admits an axis of symmetry of order 2 (cf. Schubert [8]), and rotation about this axis induces an outer automorphism of $\pi L$, viz. $x_{1} \mapsto x_{2} \mapsto x_{1}$. Hence

$$
\hat{w} x_{2}=x_{2} \hat{w} \text {, where } \hat{w}=x_{1}^{\varepsilon_{1}} x_{2}^{\varepsilon_{2}} x_{1}^{\varepsilon_{3}} \cdots x_{1}^{\varepsilon_{n-1}} .
$$

Following the proof of Lemma 2 below we give an example of a limk group in which (2.2) does not hold.

Our business with 2-bridge limk groups is representing them in $\mathrm{SL}_{2}(R), R$ an appropriate integral domain. Because the generators $x_{1}, x_{2}$ of $\pi L$ are not conjugate in $\pi L$ we have to allow for $\operatorname{tr}\left(x_{1} \phi\right) \neq \operatorname{tr}\left(x_{2} \phi\right)$, but otherwise the matrices (1.9) would do. So we now use $C=C\{t\}$ and $D=D\{s, u\}$ over $R=\mathbb{Z}\left[t, t^{-1}, s, s^{-1}, u\right]$. Write

$$
W=w(C, D)=\left[\begin{array}{cc}
w_{11} & w_{12} \\
u w_{21} & w_{22}
\end{array}\right], \quad w_{i j}=w_{i j}(t, s, u) .
$$


(The 2, 1 entry of any word on $C, D$ is a multiple of $u$, and for links there are advantages in exhibiting this notationally.) We now require that $W C=C W$. This matrix equation is easily seen to be equivalent to two entry equations:

$$
\begin{gathered}
u w_{21}=0 \\
\left(t-t^{-1}\right) w_{12}+w_{22}-w_{11}=0 .
\end{gathered}
$$

Lemma 1. Let $W$ be one of $D^{\varepsilon_{1}} C^{\varepsilon_{2}} D^{\varepsilon_{3}} \ldots D^{\varepsilon_{n}}, C^{\varepsilon_{1}} D^{\varepsilon_{2}} C^{\varepsilon_{3}} \cdots C^{\varepsilon_{n}}$, where $n$ is odd and $\vec{\varepsilon}$ is palindromic. Then

$$
\left(t-t^{-1}\right) w_{12}+w_{22}-w_{11} \equiv\left(s-s^{-1}\right) w_{21} .
$$

Hence $w_{21}=0$ alone determines the nonabelian representations of a 2-bridge limk group.

Proof. Remark first that (2.5) holds for $W$ if and only if it holds for $W^{-1}$. Our proof is a combination of this remark with induction. When $n=1$ and $W=C$ or $D$ we get $(2.5)$ directly. Next, if (2.5) holds for $W$, then one calculates that it also holds for $C W C$ and $D W D$. All palindromic words on $C, D$ are built up by this process and by taking inverses, so Lemma 1 is proved.

We call $\Phi_{\pi L}(t, s, u)=\Phi(t, s, u)=\Phi=w_{21}$ the nab-representation polynomial for the limk group $\pi L$. The equation $\Phi=0$ defines an affine algebraic surface in $\mathbb{C}^{3}$ (or in $F^{3}$ where $F$ is any field), which may be reducible. This polynomial and surface are worthy of further investigation, but we prove only what we need for $\S \S 4-6$.

Lemma 2. The polynomial $\Phi_{\pi L}(t, s, u)$ of a 2-bridge limk group has no repeated factors of positive degree in $u$. Consequently, if $\pi L$ is actually a 2-bridge link group then $\Phi_{\pi L}(t, s, u)=\Phi_{\pi L}(s, t, u)$.

Proof. As in the proof of Lemma 2 of [7], we see that $\operatorname{deg}_{u} \Phi=(\alpha-2) / 2=m$ say, and that the coefficient of $u^{m}$ in $\Phi$ is \pm 1 . If $\Phi_{\pi L}(t, s, u)$ has a repeated factor of positive degree in $u$ then $\Phi_{\pi L}(i, i, u)$ will too, where $i=\sqrt{-1}$, and then there would be fewer than $m$ distinct roots of $\Phi(i, i, u)=0$. We exclude the possibility of having a repeated factor by exhibiting $m$ distinct roots. Let $C=C\{i\}$ and $D=D\{i, u\}$; then $C^{-1}=-C$ and $D^{-1}=-D$ in $\mathrm{SL}_{2}$. This leads to

$$
W=\varepsilon_{\alpha / 2} D(C D)^{m}, \quad W^{-1}=-W, \quad W C W^{-1} C^{-1}=(D C)^{\alpha} \in \mathrm{SL}_{2} .
$$

If $u$ is chosen so that $(D C)^{\alpha}=E$ then $x_{1} \mapsto C, x_{2} \mapsto D$ is a nonabelian representation of $\pi L$ and hence $\Phi_{\pi L}(i, i, u)=0$. Now $\operatorname{tr}(D C)=-2-u$ determines the order of $D C$ in $\mathrm{SL}_{2}$, and if we set $\operatorname{tr}(D C)=2 \cos k \pi /(m+1)$ for some $k \in\{1, \ldots, m\}$ we get $(D C)^{\alpha}=E$. Hence the $m$ numbers $-2-$ $2 \cos k \pi /(m+1)$ are distinct roots of $\Phi(i, i, u)=0$, and the first assertion of Lemma 2 is proved. We note that all these roots lie in the open real interval $(-4,0)$, so that $u+4 \neq 0$ when $\Phi_{\pi L}(i, i, u)=0$.

Suppose now $\pi L$ is actually a link group, then relation (2.2) also holds in $\pi L$. Therefore if $\Phi_{\pi L}(t, s, u)=0$ then $\widehat{W} D=D \widehat{W}$, where $\widehat{W}=\hat{w}(C, D)$. We are about to invoke part A of Lemma 5 of $\S 3$ with its $\zeta=u-\left(s^{-1}-s\right)\left(t^{-1}-t\right)$. We have $\zeta(i, i, u)=u+4 \neq 0$ when $\Phi(i, i, u)=0$, so $\zeta(t, s, u) \nmid \Phi(t, s, u)$. 
Hence there are at most finitely many curves in $\mathbb{C}^{3}$ where $\zeta=0=\Phi$. Except along these curves, Lemma 5 delivers $P_{A} \in \mathrm{SL}_{2}(\mathbb{C})$ such that

$$
\begin{aligned}
P_{A} \widehat{W}(t, s, u) P_{A}^{-1} & =P_{A} C\{t\}^{\varepsilon_{1}} D\{s, u\}^{\varepsilon_{2}} \cdots C\{t\}^{\varepsilon_{\alpha-1}} P_{A}^{-1} \\
& =D\{t, u\}^{\varepsilon_{1}} C\{s\}^{\varepsilon_{2}} \cdots D\{t, u\}^{\varepsilon_{\alpha-1}}=W(s, t, u) .
\end{aligned}
$$

So conjugating $\widehat{W} D=D \widehat{W}$ by $P_{A}$ produces $W(s, t, u) C\{s\}=C\{s\} W(s, t, u)$, a relation which holds if and only if $\Phi_{\pi L}(s, t, u)=0$. This implies that each irreducible factor of $\Phi(t, s, u)$ (in $F\left[t, t^{-1}, s, s^{-1}, u\right], F$ a field) divides $\Phi_{\pi L}(s, t, u)$. Because there are no repeated factors, $\Phi(t, s, u)$ divides $\Phi(s, t, u)$. This relation is symmetric, and so $\Phi(s, t, u)=z \cdot \Phi(t, s, u)$, where $\operatorname{deg}_{u} z=0$. But the coefficient of the highest power of $u$ in $\Phi$ is an integer independent of $s$ or $t$, whence $\Phi(t, s, u)=\Phi(s, t, u)$. Lemma 2 is proved.

Calculation shows that $\Phi(t, s, u) \neq \Phi(s, t, u)$ for the limk group $\pi L$ of determinant 6 whose exponent sequence is $(1,1,-1,1,1)$. Consequently (2.2) does not hold in $\pi L$.

W. Thurston in [9] showed that a link $l \subset S^{3}$ whose complement admits the excellent hyperbolic structure has a representation variety of dimension $\geq c(l)$, where $c(l)$ is the number of components of $l$. (We are speaking of simple equivalence classes of nonabelian representations; add 3 to get the dimension of the variety of all such representations.) So when the 2-bridge link $L$ is not a torus link $(\alpha, 1), \Phi_{\pi L}$ has an "excellent" factor $\Phi_{\mathrm{ex}}(t, s, u)$ such that for some $u_{1} \in \mathbb{C}, \Phi_{\mathrm{ex}}\left(1,1, u_{1}\right)$ corresponds to a parabolic representation $\theta: \pi L \rightarrow \mathrm{SL}_{2}(\mathbb{C})$ which is faithful and has discrete image. It follows that the representations for generic points on $\Phi_{\text {ex }}(t, s, u)=0$ or on $\Phi_{\text {ex }}(t, t, u)=0$ are faithful. A similar argument for the 2-bridge torus links produces a "good" factor of $\Phi_{\pi L}$ whose generic representations have kernel $=\operatorname{centre}(\pi L)$.

\section{MatriX identities}

The material of this section is widely known in various versions, but a proper ascription of priority would be difficult. A search for precedents should start with Euler since he may have published the substance of these identities in different notation.

Lemma 3. Let $A, B$ be in $M_{2}(R)$, where $R$ is an integral domain. Then

$$
A B A=\operatorname{tr}(A B) A-\operatorname{det}(A) \operatorname{adj}(B) .
$$

Proof. Introduce the polynomial domain $R[x]$ with quotient field $F(x)$, and consider $B_{x}=B+x E \in M_{2}(R[x])$. Then $B_{x}$ is invertible in $M_{2}(F(x))$, because $\operatorname{det}\left(B_{x}\right) \in R[x]$ is not the zero polynomial. The Cayley-Hamilton theorem applied to $A B_{x}$ yields

$$
A B_{x} A \cdot B_{x}=\operatorname{tr}\left(A B_{x}\right) A \cdot B_{x}-\operatorname{det}\left(A B_{x}\right) E .
$$

Multiply through on the right by $B_{x}^{-1}$ and use $\operatorname{adj}\left(B_{x}\right)=\operatorname{det}\left(B_{x}\right) B_{x}^{-1}$ to get (3.1) for $A, B_{x}$. But this is an identity in $M_{2}(R[x])$, and therefore we can set $x=0$ in it to get (3.1) on $A, B$. 
A sequence of polynomials $\left\{\tau_{n}(x)\right\}$ in $\mathbb{Z}[x]$ closely related to the MorganVoyce polynomials is defined by

(3.2) $\tau_{0} \equiv 0, \quad \tau_{1} \equiv 1, \quad \tau_{n+1}(x)=x \tau_{n}(x)-\tau_{n-1}(x)$ for $n \geq 1, \quad \tau_{-n}=-\tau_{n}$.

These are used to express the powers of a unimodular matrix $M \in \mathrm{SL}_{2}(R), R$ an integral domain, as linear combinations of $M$ and $E$, namely

$$
M^{n}=\tau_{n}(\sigma) M-\tau_{n-1}(\sigma) E, \quad \sigma=\operatorname{tr}(M) .
$$

The proof is an obvious induction. If we take $M$ to be the defining matrix for the Morgan-Voyce polynomials we can compare the two sorts:

$$
\left[\begin{array}{cc}
f_{n}(x) & g_{n-1}(x) \\
x g_{n-1}(x) & f_{n-1}(x)
\end{array}\right]=\left[\begin{array}{cc}
1+x & 1 \\
x & 1
\end{array}\right]^{n}=\left[\begin{array}{cc}
(1+x) \tau_{n}-\tau_{n-1} & \tau_{n} \\
x \tau_{n} & \tau_{n}-\tau_{n-1}
\end{array}\right]
$$

where $\tau_{\nu}=\tau_{\nu}(2+x)$. This gives

$$
\tau_{n}(2+x)=g_{n-1}(x) \text { and }(1+x) \tau_{n}(2+x)-\tau_{n-1}(2+x)=f_{n}(x) .
$$

Proposition 2. Let $A, B \in \mathrm{SL}_{2}(R)$, where $R$ is an integral domain. Then for $n \geq 0$,

$$
\left(A B^{-1}\right)^{n} A=f_{n}(v) A+g_{n-1}(v)(A-B),
$$

where $v=\operatorname{tr}\left(A B^{-1}\right)-2$.

Proof. Because $A$ and $B$ are unimodular, (3.1) on $A, B^{-1}$ becomes

$$
A B^{-1} A=(2+v) A-B=(1+v) A+(A-B) .
$$

Also $\left(A B^{-1}\right)^{n}=\tau_{n}(2+v) A B^{-1}-\tau_{n-1}(2+v) E$, so

$$
\begin{aligned}
\left(A B^{-1}\right)^{n} A & =\tau_{n}(2+v) A B^{-1} A-\tau_{n-1}(2+v) A \\
& =(1+v) \tau_{n}(2+v) A+\tau_{n}(2+v)(A-B)-\tau_{n-1}(2+v) A \\
& =f_{n}(v) A+g_{n-1}(v)(A-B),
\end{aligned}
$$

proving Proposition 2.

The Morgan-Voyce polynomials $f_{n}(x)$ and $g_{n}(x)$ for $n \geq 1$ have all their roots in the real interval $-4<x<0$ (cf. [4, 5]).

Lemma 4. Let $x_{n}<x_{n-1}<\cdots<x_{1}$ be the roots of $g_{n}(x)=0$. Then

Proof. We have

$$
f_{n}\left(x_{k}\right)=(-1)^{k}, \quad k=1, \ldots, n .
$$

$$
\left[\begin{array}{cc}
1+x & 1 \\
x & 1
\end{array}\right]^{n+1}=\left[\begin{array}{cc}
f_{n}+x g_{n} & g_{n} \\
x g_{n} & f_{n}
\end{array}\right], \quad f_{n}=f_{n}(x), \quad g_{n}=g_{n}(x),
$$

so if $g_{n}(x)=0$ this becomes $\operatorname{diag}\left(f_{n}, f_{n}\right)$. But the determinant is 1 , whence $f_{n}\left(x_{k}\right)= \pm 1$ for all $x_{k}$. To settle the sign we write $y_{n}<y_{n-1}<\cdots<y_{1}$ for the roots of $f_{n}(x)=0$, and use the explicit formulae for $\left\{x_{k}\right\}$ and $\left\{y_{k}\right\}$ from $[4,5]$ :

$$
\left.\begin{array}{rl}
x_{k} & =-\left(2 \sin \frac{\pi}{2} \frac{k}{n+1}\right)^{2} \\
y_{k} & =-\left(2 \cos \frac{\pi(n+1-k)}{2 n+1}\right)^{2} \\
& =-\left(2 \sin \frac{\pi}{2} \frac{2 k-1}{2 n+1}\right)^{2}
\end{array}\right\}, \quad k=1, \ldots, n .
$$


Then $\left|y_{k}\right|<\left|x_{k}\right|<\left|y_{k+1}\right|$ follows from the elementary inequalities

$$
\frac{2 k-1}{2 n+1}<\frac{k}{n+1}<\frac{2 k+1}{2 n+1} \text {. }
$$

Also $f_{n}(0)=1$, so $x_{1}$ lies in an interval where $f_{n}(x)$ is negative, and the rest is clear.

The matrices

$$
C=C\{t\}=\left[\begin{array}{cc}
t & 1 \\
0 & t^{-1}
\end{array}\right], \quad D=D\{s, u\}=\left[\begin{array}{cc}
s & 0 \\
-u & s^{-1}
\end{array}\right]
$$

belong to $\mathrm{SL}_{2}(R)$, where $R=\mathbb{Z}\left[t, t^{-1}, s, s^{-1}, u\right]$. Let $F=\mathbb{Q}(t, s, u)$ be the quotient field of $R$ and let $F_{\infty}=F \cup\{\infty\}$. Then $C$ and $D$ act as fractional linear transformations of $F_{\infty}$ by the usual rule: $x \mapsto(a x+b) /(c x+d)$. As such, $C$ fixes $\infty,\left(t^{-1}-t\right)^{-1}$, and $D$ fixes $0, u^{-1}\left(s^{-1}-s\right)$. A polynomial equation in $R$ which is equivalent to the assertion that $C$ and $D$ have a common fixed point in $F_{\infty}$ is

$$
u \cdot \zeta=0, \quad \zeta=u-\left(s^{-1}-s\right)\left(t^{-1}-t\right) .
$$

Lemma 5. A. There exists $P_{A} \in \mathrm{SL}_{2}(F(\sqrt{\zeta}))$ such that

$$
P_{A} C\{t\} P_{A}^{-1}=D\{t, u\}, \quad P_{A} D\{s, u\} P_{A}^{-1}=C\{s\} .
$$

B. There exists $P_{B} \in \mathrm{SL}_{2}(F(\sqrt{\zeta}))$ such that

$$
P_{B} \cdot C\{t\}=C\{t\} \cdot P_{B}, \quad P_{B} \cdot D\{s, u\}^{-1} \cdot P_{B}^{-1}=D\{s, \tilde{u}\}
$$

if (and only if) $\tilde{u}=-\zeta$.

C. (T. Jorgensen [2]) There exists $P_{C} \in \mathrm{SL}_{2}(F(\sqrt{-u \zeta}))$ such that

$P_{C}^{2}=-E, \quad P_{C} \cdot C\{t\} \cdot P_{C}^{-1}=C\{t\}^{-1}, \quad P_{C} \cdot D\{s, u\} \cdot P_{C}^{-1}=D\{s, u\}^{-1}$.

Proof. It suffices to exhibit the explicit matrices $P_{A}, P_{B}, P_{C}$, so that the verifications become an exercise:

$$
\begin{aligned}
P_{A} & =\frac{1}{\sqrt{\zeta}}\left[\begin{array}{cc}
t-t^{-1} & 1 \\
-u & s^{-1}-s
\end{array}\right], \quad P_{B}=\frac{1}{\sqrt{u \zeta}}\left[\begin{array}{cc}
u & s-s^{-1} \\
0 & \zeta
\end{array}\right], \\
P_{C}=\frac{1}{\sqrt{-u \zeta}}(C D-D C), \quad C D-D C & =\left[\begin{array}{cc}
-u & s^{-1}-s \\
\left(t-t^{-1}\right) u & u
\end{array}\right] .
\end{aligned}
$$

This completes the proof.

We remark that if $t, s, u$ are taken to be specific numbers in a field then none of $P_{A}, P_{B}, P_{C}$ exist if $\zeta(s, t, u)=0$, and only $P_{A}$ exists if $u=0 \neq \zeta$.

\section{HeCKOID ALGEBRA FOR 2-BRIDGE KNOTS AND LINKS}

We begin with kmot groups. Let $\pi K$ be a kmot group presented by (1.1), and let $W=w(C, D), \Phi=\Phi_{\pi K}(t, u)$, and $R=\mathbb{Z}\left[t, t^{-1}, u\right]$, using (1.9)-(1.11). For $e= \pm 1$,

$$
\begin{gathered}
W C^{e}=\left[\begin{array}{cc}
t^{e} w_{11} & t^{-e} w_{12}+e w_{11} \\
-t^{e} u w_{12} & t^{-e} w_{22}-e u w_{12}
\end{array}\right] \\
t^{e} w_{11} \\
D^{e} W=\left[\begin{array}{cc}
t^{e} w_{12} \\
-u\left(e w_{11}+t^{-e} w_{12}\right) & t^{-e} w_{22}-e u w_{12}
\end{array}\right], \\
V_{e}=W C^{e}\left(D^{e} W\right)^{-1}=\left[\begin{array}{cc}
1+u\left(w_{11}+e t^{-e} w_{12}\right) \Phi & e t^{e} w_{11} \Phi \\
u\left(-u w_{12}+e t^{-e} w_{22}\right) \Phi & 1-e t^{e} u w_{12} \Phi
\end{array}\right] .
\end{gathered}
$$


It was clear in advance that $V_{e} \equiv E(\bmod \Phi)$, but we seem to need a calculation to determine its trace. We get

$$
\operatorname{tr}\left(V_{e}\right)=2+u \Phi^{2}=2+v, \quad \text { where } v=v_{\pi K}=u \Phi^{2}
$$

We also write $\bar{V}_{e}=\bar{W} C^{-e}\left(D^{-e} \bar{W}\right)^{-1}$, where $\bar{W}=w\left(C^{-1}, D^{-1}\right)=\bar{w}(C, D)$ as in (1.3). The above calculations can also be made for the trivial knot $O$, using $w=E, \Phi=1$, to get $v=u$. We tacitly include $O$ with the 2-bridge knots in Theorem 1 with these values.

Theorem 1. For each 2-bridge kmot group the following are true.

(i) For integral $n \geq 1$ let $q=2 n+1, U_{e, n}=V_{e}^{n} W C^{e}$, and $\bar{U}_{e, n}=$ $\bar{V}_{e}^{n} \bar{W} C^{-e}$. Also let $C^{\delta_{1}} D^{\delta_{2}} C^{\delta_{3}} \ldots C^{\delta_{q \alpha}}$ be the formal representation of $U_{e, n}$ as a word on $C, D$. Then in $\mathrm{SL}_{2}\left(R /\left(f_{n}(v)\right)\right)$ one has

$$
\begin{gathered}
U_{1, n}=-U_{-1, n}, \quad U_{e, n}^{2}=V_{e}^{q}=\bar{V}_{e}^{q}=-E, \quad U_{e, n} C=D^{-1} U_{e, n}, \\
U_{e, n}=-\bar{U}_{e, n}=D^{-\delta_{1}} C^{-\delta_{2}} D^{-\delta_{3}} \cdots D^{-\delta_{q \alpha}}=-D^{\delta_{1}} C^{\delta_{2}} D^{\delta_{3}} \cdots D^{\delta_{q \alpha}}
\end{gathered}
$$

(ii) For integral $n \geq 1$ let $q=2 n+2$. Then in $\operatorname{SL}_{2}\left(R /\left(g_{n}(v)\right)\right)$ one has $V_{e}^{q}=\bar{V}_{e}^{q}=E$. More exactly, if $(t, u) \in \mathbb{C} \times \mathbb{C}$ lies on the locus $g_{n}(v)=0$, then for some $k$, where $1 \leq k \leq n$, one has $v(t, u)=x_{k}$ of Lemma 4, and

$$
V_{e}^{n+1}(t, u)=\bar{V}_{e}^{n+1}(t, u)=(-1)^{k} E \quad \text { in } \mathrm{SL}_{2}(\mathbb{C}) \text {. }
$$

Proof. We consider (ii) first because it is needed for (i). Write $\tau_{\nu}=\tau_{\nu}(2+v)=$ $g_{\nu-1}(v)$ for $\nu=n, n+1$. When $v(t, u)=x_{k}$ we have

$$
V_{e}^{n+1}=\tau_{n+1} \cdot V_{e}-\tau_{n} \cdot E=g_{n}\left(x_{k}\right) \cdot V_{e}-g_{n-1}\left(x_{k}\right) E=-g_{n-1}\left(x_{k}\right) E .
$$

But $0=g_{n}\left(x_{k}\right)=f_{n}\left(x_{k}\right)+g_{n-1}\left(x_{k}\right)=(-1)^{k}+g_{n-1}\left(x_{k}\right)$, whence $V_{e}^{n+1}=$ $(-1)^{k} E$. Square to get $V_{e}^{q}=E$. Lemma 5 implies $\bar{V}_{e}=P_{C} V_{e} P_{C}^{-1}$, whence $\bar{V}_{e}^{n+1}=V_{e}^{n+1}$, when $h(t, u)=u \cdot\left[\left(t-t^{-1}\right)^{2}-u\right] \neq 0$. But when $u=0$ then $v=0$, whereas $g_{n}(0)=n+1 \neq 0$. When $u=\left(t-t^{-1}\right)^{2}$ then $\Phi(t, u)=1$ by Lemma 4 of [7], whence $v=u=\left(t-t^{-1}\right)^{2}$. So $h(t, u)=g_{n}(v)=0$ leads to $g_{n}\left(\left(t-t^{-1}\right)^{2}\right)=0$, which accounts for only a finite set of points on the locus $g_{n}(v)=0$. This means that $V_{e}^{n+1}=\bar{V}_{e}^{n+1}$ on the locus $g_{n}(v)=0$, except possibly for a finite set of points, but because the matrix entries are rational functions of $t$ and $u, V_{e}^{n+1} \equiv \bar{V}_{e}^{n+1}$ on $g_{n}(v)=0$. This proves (ii).

(i) We apply Proposition 2 with $A=W C^{e}, B=D^{e} W$, using the calculations leading to the definition of $v$. We get

$$
V_{e}^{n} W C^{e}=f_{n}(v) W C^{e}+g_{n-1}(v)\left(W C^{e}-D^{e} W\right) .
$$

When $f_{n}(v)=0$ this becomes

$$
\begin{aligned}
U_{e, n} & =g_{n-1}(v)\left[\begin{array}{cc}
0 & e w_{11}+\left(t^{-e}-t^{e}\right) w_{12} \\
u\left\{e w_{11}+\left(t^{-e}-t^{e}\right) w_{12}\right\} & 0
\end{array}\right] \\
& =e \Phi g_{n-1}(v) \cdot\left[\begin{array}{ll}
0 & 1 \\
u & 0
\end{array}\right] .
\end{aligned}
$$


So $U_{1, n}=-U_{-1, n}$ in $\operatorname{SL}_{2}\left(R /\left(f_{n}(v)\right)\right)$, and from $\operatorname{det} U_{e, n}=1$ one gets $-u \Phi^{2} g_{n-1}(v)^{2}=1, \Phi g_{n-1}(v)=1 / \sqrt{-u}$. Therefore

$$
U_{e, n}=e\left[\begin{array}{cc}
0 & 1 / \sqrt{-u} \\
-\sqrt{-u} & 0
\end{array}\right] \in \mathrm{SL}_{2}\left(R /\left(f_{n}(v)\right)\right) .
$$

Then $U_{e, n} C=D^{-1} U_{e, n}$ becomes an immediate computation. When $(t, u) \in$ $\mathbb{C} \times \mathbb{C}$ satisfies $f_{n}(v)=0$ then $v(t, u)=y_{k}$ for one of the values in (3.6). By comparing the formulae for $x_{k}, y_{k}$ in (3.6) one sees that our $y_{k}$ equals $x_{2 k-1}$ of $g_{2 n}(x)=0$, and so by part (ii), $V_{e}^{q}=-E$. One deduces $V_{e}^{q}=\bar{V}_{e}^{q}$ from Lemma 5 by the argument used in part (ii) above. Direct calculation with $\Delta=C D-D C$ of (3.8) shows that $\Delta U_{e, n} \Delta^{-1}=-U_{e, n}$ when $\operatorname{det} \Delta \neq 0$, so by the same argument again, $U_{e, n}=-\bar{U}_{e, n} \in \mathrm{SL}_{2}\left(R /\left(f_{n}(v)\right)\right)$.

To get the remaining expressions for $U_{e, n}$ in $\operatorname{SL}_{2}\left(R /\left(f_{n}(v)\right)\right)$ we have

$$
\begin{aligned}
U_{e, n} & =U_{e, n} \cdot C^{\delta_{1}} D^{\delta_{2}} C^{\delta_{3}} \cdots C^{\delta_{q \alpha}} \cdot U_{e, n}^{-1} \\
& =U_{e, n} C^{\delta_{1}} U_{e, n}^{-1} \cdot U_{e, n} D^{\delta_{2}} U_{e, n}^{-1} \cdots \cdots U_{e, n} C^{\delta_{q \alpha}} U_{e, n}^{-1} \\
& =D^{-\delta_{1}} C^{-\delta_{2}} D^{-\delta_{3}} \cdots D^{-\delta_{q \alpha}} .
\end{aligned}
$$

This argument applied to $\bar{U}_{e, n}=-U_{e, n}$ produces the last formula for $U_{e, n}$. This completes the proof of Theorem 1.

Corollary. (i) In $\operatorname{PSL}_{2}\left(R /\left(f_{n}(v)\right)\right)$ with $q=2 n+1$ one has

$$
\begin{gathered}
U_{e, n}^{2}=V_{e}^{q}=\bar{V}_{e}^{q}=E, \quad U_{e, n} C=D^{-1} U_{e, n}, \\
U_{1, n}=U_{-1, n}=\bar{U}_{e, n}=D^{\delta_{1}} C^{\delta_{2}} D^{\delta_{3}} \cdots D^{\delta_{q \alpha}}=D^{-\delta_{1}} C^{-\delta_{2}} D^{-\delta_{3}} \cdots D^{-\delta_{q \alpha}} .
\end{gathered}
$$

(ii) $V_{e}^{n+1}=\bar{V}_{e}^{n+1}=E$ in $\operatorname{PSL}_{2}\left(R /\left(g_{n}(v)\right)\right)$.

The Heckoid algebra for limk groups runs along similar lines and the presence of the third parameter $s$ will make little difference. Let $\pi L$ be a 2-bridge limk group of determinant $\alpha$, presented by $(2.1)$, and let $W=w(C, D)$ and $\Phi=\Phi_{\pi L}(t, s, u)$, using $C=C\{t\}, D=D\{s, u\}$. For $e= \pm 1$ we have

$$
\begin{gathered}
W C^{e}=\left[\begin{array}{cc}
t^{e} w_{11} & e w_{11}+t^{-e} w_{12} \\
t^{e} u w_{21} & e u w_{21}+t^{-e} w_{22}
\end{array}\right], \\
C^{e} W=\left[\begin{array}{cc}
t^{e} w_{11}+e u w_{21} & t^{e} w_{12}+e w_{22} \\
t^{-e} u w_{21} & t^{-e} w_{22}
\end{array}\right],
\end{gathered}
$$

before we use (2.5), the consequence of the palindromic symmetry of $w$, to eliminate $w_{22}$, and $\operatorname{det} W=1$ to eliminate $w_{12}$. We calculate that $V_{e}:=$ $W C^{e}\left(C^{e} W\right)^{-1}$ has trace $\operatorname{tr}\left(V_{e}\right)=2+v$, where $v=v_{L}(t, s, u)$ is given by

$$
v=u\left[u-\left(s^{-1}-s\right)\left(t^{-1}-t\right)\right] w_{21}^{2}=u \zeta \Phi_{\pi L}^{2} .
$$

(It suffices to do this directly just for $e=+1$, because trace identities imply $\operatorname{tr}\left(V_{1}\right)=\operatorname{tr}\left(V_{-1}\right)$.) Let $\bar{V}_{e}:=W^{-1} C^{-e}\left(C^{-e} W^{-1}\right)^{-1}$, i.e., $\bar{V}_{e}$ is the word $V_{e}$ on $C, D$ with all exponents negated. We write $\mathbb{Z}\left[t, t^{-1}, s, s^{-1}, u\right]=R$ for the rest of this section. 
Theorem 2. For each 2-bridge limk group the following are true.

(i) For $n \geq 1$ let $q=2 n+1, U_{e, n}=V_{e}^{n} W C^{e}$, and $\bar{U}_{e, n}=\bar{V}_{e}^{n} W^{-1} C^{-e}$. Then in $\mathrm{SL}_{2}\left(R /\left(f_{n}(v)\right)\right)$ we have

$$
\begin{array}{cl}
U_{1, n}=-U_{-1, n}=\bar{U}_{e, n}, & U_{e, n}^{2}=V_{e}^{q}=\bar{V}_{e}^{q}=-E, \\
U_{e, n} C U_{e, n}^{-1}=C^{-1}, & U_{e, n} D U_{e, n}^{-1}=D^{-1} .
\end{array}
$$

(ii) For $n \geq 1$ let $q=2 n+2$. Then $V_{e}^{q}=\bar{V}_{e}^{q}=E$ in $\mathrm{SL}_{2}\left(R /\left(g_{n}(v)\right)\right)$. More exactly, if $v(t, s, u)=x_{k}$ of Lemma 4 then $V_{e}^{n+1}=\bar{V}_{e}^{n+1}=(-1)^{k} E$ in $\mathrm{SL}_{2}(\mathbb{C})$.

Proof. Part (ii) here has nearly the same proof as part (ii) of Theorem 1. This time the coefficient of $w_{21}$ in $v$ is the negative of $\operatorname{det}(\Delta), \Delta=C D-D C$, so one can find the inverting matrix $P_{C}$ of Lemma 5 except when $v=0$, in which event $g_{n}(v) \neq 0$. The proof of part (i) is also so similar to that for Theorem 1 that we need give only highlights. We apply (2.4) to $W C^{e}-C^{e} W$ to get

$$
W C^{e}-C^{e} W=e w_{21}\left[\begin{array}{cc}
-u & s^{-1}-s \\
\left(t-t^{-1}\right) u & u
\end{array}\right]=e w_{21} \Delta .
$$

Now Proposition 2 with $A=W C^{e}, B=C^{e} W$ leads to

$$
U_{e, n}=\left[W C^{e}\left(C^{e} W\right)^{-1}\right]^{n} W C^{e}=f_{n}(v) W C^{e}+g_{n-1}(v)\left(W C^{e}-C^{e} W\right) .
$$

Over $R /\left(f_{n}(v)\right)$ this is the second term alone, and as $v=0, f_{n}(v)=1$ when $\operatorname{det} \Delta=0$, $\operatorname{det} \Delta \neq 0$ in $R /\left(f_{n}(v)\right)$. So $\operatorname{det} U_{e, n}=1$ leads to

$$
U_{e, n}=\frac{e w_{21}}{\sqrt{-v}}\left[\begin{array}{cc}
-u & s^{-1}-s \\
\left(t-t^{-1}\right) u & u
\end{array}\right]=\frac{e}{\sqrt{\operatorname{det} \Delta}} \Delta .
$$

Thus $U_{e, n}$ inverts $C$ and $D$ by (3.8), whence conjugating $U_{e, n}$ by $U_{e, n}$ produces $\bar{U}_{e, n}=U_{e, n}$. The rest of the proof is the same as before.

Corollary. (i) In $\operatorname{PSL}_{2}\left(R /\left(f_{n}(v)\right)\right)$ with $q=2 n+1$ one has

$$
U_{1, n}=U_{-1, n}=\bar{U}_{e, n}, \quad U_{1, n}^{2}=V_{e}^{q}=\bar{V}_{e}^{q}=E,
$$

and conjugation by $U_{1, n}$ inverts $C$ and $D$.

(ii) $V_{e}^{n+1}=\bar{V}_{e}^{n+1}=E$ in $\operatorname{PSL}_{2}\left(R /\left(g_{n}(v)\right)\right)$.

Lemma 6. When $X \in\langle C\{t\}, D\{s, u\}\rangle$ is a formal word on $C$ and $D$ let $J(X)$ be the word obtained by replacing each $C$ in $X$ by $D$ and each $D$ by $C$. If $\pi L$ is a 2-bridge link group then the relations of Theorem 2 on $V_{e}, \bar{V}_{e}, U_{e, n}$, $\bar{U}_{e, n}$ in $\mathrm{SL}_{2}(R /(h))$, where $h=f_{n}(v)$ or $h=g_{n}(v)$, hold in the same quotient rings when we replace these words $X$ by their associates $J(X)$.

Proof. When $X$ is one of $V_{e}, \ldots, \bar{U}_{e, n}$ write $X=D^{\gamma(1)} C^{\gamma(2)} \ldots$. Then $J(X)=C^{\gamma(1)} D^{\gamma(2)} \ldots$. We conjugate by the matrix $P_{A}$ of Lemma 5 (assuming it exists):

$$
P_{A} J(X) P_{A}^{-1}=D\{t, u\}^{\gamma(1)} C\{s\}^{\gamma(2)} \cdots .
$$

Hence if $\operatorname{Rel}(X, Y, \ldots)(t, s, u)$ is one of the relations of Theorem 2 on $V_{e}, \ldots, \bar{U}_{e, n}$ we get

$$
P_{A} \operatorname{Rel}(J(X), J(Y), \ldots)(t, s, u) P_{A}^{-1}=\operatorname{Rel}(X, Y, \ldots)(s, t, u) .
$$


Hence these relations hold in $R /(h(s, t, u))$ by Theorem 2. But $h(t, s, u)=$ $f_{n}(v)$ or $g_{n}(v)$, and $v=u \zeta(t, s, u) \Phi_{\pi L}^{2}(t, s, u)$. Now $\zeta$ is visibly symmetric in $s$ and $t$, and because $\pi L$ is actually a link group, Lemma 2 tells us that $\Phi$ is also symmetric, so $v$ is too. Therefore $h(t, s, u)=h(s, t, u)$, and the conclusions of Lemma 6 do hold provided $P_{A}$ exists on the loci $f_{n}(v)=0$, $g_{n}(v)=0$. But $P_{A}$ exists except when $\zeta=0$, and if $\zeta=0$ then $v=0$. However, $f_{n}(0)=1$ and $g_{n}(0)=n+1$, completing the proof.

\section{EXPONENT SEQUENCES FOR CERTAIN 2-BRIDGE KNOTS AND LINKS}

From now on our results are restricted to genuine knots and links, although perhaps versions for proper kmot and limk groups can be found. Now that we have some experience about the similarities and differences of the knot and link cases we shall try to give a unified account.

Let $K=(\alpha, \beta)$ be a 2-bridge knot normal form. Then $\alpha \beta$ is odd, $\operatorname{gcd}(\alpha, \beta)$ $=1$, and $|\beta|<2 \alpha$. Because we are only interested in the knot group $\pi K$ we can and shall assume $0<\beta<\alpha$. We again include the trivial knot $O=(1,1)$ (where now $\alpha=\beta=1$ ). We use the standard presentation (1.1) of $\pi K$ with its conjugating word $w=w\{\alpha, \beta\}$ which has exponent sequence $\vec{\varepsilon}=$ $\left(\varepsilon_{1}, \ldots, \varepsilon_{\alpha-1}\right)$. Associated with $K$ is the derived pair $\widetilde{K}=(\alpha, \tilde{\beta})$, where $\tilde{\beta}=$ $\alpha-\beta$. Because $\tilde{\beta}$ is even, $\widetilde{K}$ is not a 2 -bridge normal form, but nevertheless a modified version of (1.1), (1.2) can be applied to produce a presentation of a group

$$
\begin{array}{rlrl}
\pi \widetilde{K} & =\left|x_{1}, x_{2}: \tilde{w} x_{1}=x_{2}^{-1} \tilde{w}\right|, & \tilde{w} & =x_{1}^{\tilde{\varepsilon}_{1}} x_{2}^{\tilde{\varepsilon}_{2}} x_{1}^{\tilde{\varepsilon}_{3}} \cdots x_{2}^{\tilde{\varepsilon}_{\alpha-1}}, \\
\tilde{\varepsilon}_{k}=(-1)^{k+1} \varepsilon_{k}, \quad \tilde{\varepsilon}_{\alpha-k}=-\tilde{\varepsilon}_{k}, & k & =1, \ldots, \alpha-1 .
\end{array}
$$

This exponent sequence with its skew palindromic symmetry is the direct result of the rule (1.2). Actually, $\pi \widetilde{K}$ is only $\pi K$ presented on $x_{1}, x_{2}^{-1}$, where $x_{1}$, $x_{2}$ are the standard meridian generators of $(1.1)$ for $\pi K$, with $x_{2}$ renamed to $x_{2}^{-1}$. The introduction of $\widetilde{K}$ facilitates comparisons between exponent sequences in the proofs below, and we get rid of it at the end with Lemma 5. (Remark that $\pi \widetilde{K}$ algebraically simulates reversing the orientation of the second bridge of a model knot for $K$, while keeping the orientation of the rest unchanged. This is the third time we have found use for this idea, the other two applications of it were to skew unions of knots [4, II].)

For a 2-bridge link normal form $L=(\alpha, \beta)$ one has $\alpha$ even, $\operatorname{gcd}(\alpha, \beta)=1$, and $|\beta|<2 \alpha$. As before we restrict to the case $0<\beta<\alpha$. This time the pair $\widetilde{L}=(\alpha, \tilde{\beta}), \tilde{\beta}=\alpha-\beta$, is a good 2-bridge link normal form, because reversing the orientation of one component of a link is not a peculiar operation. We use the standard presentation (2.1) for $\pi L$ and $\pi \widetilde{L}$, with the words $w=w\{\alpha, \beta\}$, $\tilde{w}=w\{\alpha, \tilde{\beta}\}$ and their exponent sequences $\vec{\varepsilon}, \overrightarrow{\tilde{\varepsilon}}$. The relation between $\vec{\varepsilon}$ and $\overrightarrow{\tilde{\varepsilon}}$ is now $\tilde{\varepsilon}_{k}=(-1)^{k} \varepsilon_{k}$.

From our 2-bridge normal form $K=(\alpha, \beta)$ (here " $K$ " stands for "knot or link," but we shall use " $L$ " when we know we have a link) we derive associated normal forms and pairs as follows. We use integers $e, r$, and $n$, where $e$ is +1 or $-1, n \geq 1$, and $r \geq 2$. Set $q=2 n+1$ or $q=2 n+2$, and $A=\alpha^{r-1} q, \quad \alpha^{*}=\alpha A=\alpha^{r} q, \quad \beta_{e}^{*}=A \cdot(\alpha-\beta)+e, \quad \tilde{\beta}_{e}^{*}=\alpha^{*}-\beta_{e}^{*}=A \beta-e$, 


$$
K_{e}^{*}=K^{*}(e)=\left(\alpha^{*}, \beta_{e}^{*}\right), \quad \widetilde{K}_{e}^{*}=\widetilde{K}^{*}(e)=\left(\alpha^{*}, \tilde{\beta}_{e}^{*}\right) .
$$

If $\alpha$ or $q$ is known to be even we write $L^{*}(e), \widetilde{L}^{*}(e)$ instead. The knot types of $K^{*}(1)$ and $K^{*}(-1)$ are the same, so $\Phi_{K^{*}(1)}$ and $\Phi_{K^{*}(-1)}$ have parallel factorizations, but in our experience $\Phi_{K^{*}(1)}=\Phi_{K^{*}(-1)}$ only when $K=(1,1)$.

We are about to produce two lemmas comparing the exponent sequence $\vec{\varepsilon}$ of $K$ with the sequence $\overrightarrow{\tilde{\varepsilon}}^{*}$ for $\widetilde{K}^{*}(e)$, and we often write $\varepsilon(k)$ for $\varepsilon_{k}$ in such a sequence so we can see it better. First of all, we extend $\overrightarrow{\tilde{\varepsilon}}^{*}$ to a sequence $\vec{\delta}=\left(\overrightarrow{\tilde{\varepsilon}}^{*},(-1)^{A-1} e\right)$ of length $\alpha^{*}$, i.e., $\delta(k)=\tilde{\varepsilon}^{*}(k)$ when $k<\alpha^{*}, \delta\left(\alpha^{*}\right)=$ $(-1)^{A-1} e$. This leads to the rule

$$
\delta_{k}=(-1)^{p} \text { when } k<\alpha^{*} \text { and } p \alpha^{*}<k \tilde{\beta}_{e}^{*}<(p+1) \alpha^{*} .
$$

For $l=1,2, \ldots, \alpha$, let $I(l)$ denote the sequence $(\nu, \nu+1, \ldots, l A)$, where $\nu=\nu(l)=(l-1) A+1$.

Lemma 7. Suppose $k$ is in $I(l)$ for some $l, 1 \leq l \leq \alpha$. When $k \beta=p \alpha+h$, where $l-\alpha \leq e h \leq l-1$, we have $\delta(k)=e \cdot(-1)^{p-1}$.

Proof. One has

$$
\begin{aligned}
k \tilde{\beta}_{e}^{*} & =A \cdot k \beta-k e=p \alpha^{*}+A h-k e \\
& =p \alpha^{*}+e \cdot(e h-(l-1)) A-e \cdot(k-(l-1) A) .
\end{aligned}
$$

The bounds on $h$ imply that $k \tilde{\beta}_{e}^{*}$ is strictly between $p \alpha^{*}$ and $(p-e) \alpha^{*}$, except in the extreme case where $e h=l-\alpha$ and $k=l A$, where one gets $k \tilde{\beta}_{e}^{*}=(p-e) \alpha^{*}$. In the nonextreme case, $\delta_{k}=(-1)^{p}$ when $e=-1$, and $\delta_{k}=(-1)^{p-1}$ when $e=+1$, and these values agree with $e \cdot(-1)^{p-1}$. In the extreme case, $k=l A$ implies $\alpha \mid k, h=0$, so $l=\alpha$ and $k=\alpha^{*}$. But we specifically defined $\delta\left(\alpha^{*}\right)$ to make this come out: here $p=A \beta$ has the same parity as $A$, whence $e \cdot(-1)^{p-1}=e \cdot(-1)^{A-1}$. This completes the proof of Lemma 7.

The exponent sequence $\vec{\varepsilon}$ of $K=(\alpha, \beta)$ can be extended to a sequence, still called $\vec{\varepsilon}$, of arbitrary length by

$$
\varepsilon(k)=(-1)^{p} \text { when }\left\{\begin{array}{l}
e=-1 \text { and } p \alpha \leq k \beta<(p+1) \alpha, \\
e=+1 \text { and } p \alpha<k \beta \leq(p+1) \alpha .
\end{array}\right.
$$

Note that this rule leads to $\varepsilon(k+\alpha)=-\varepsilon(k)$.

Lemma 8. For $l \in\{1, \ldots, \alpha\}$ there are integers $\mu$ and $c$, which depend only on $\alpha, \beta, e$, and $l$, such that

$$
|\mu|<\alpha \quad \text { and } \quad \delta(k)=(-1)^{c} \varepsilon(k-\mu) \quad \text { for } k \in I(l) .
$$

If $\alpha$ is odd we can choose the parity of $\mu$, and if $\alpha$ is even we can choose the sign of $\mu$.

Proof. We try first the pair $\mu, c$ determined by $0 \leq \mu<\alpha$ and $\mu \beta=c \alpha+e$. $(l-1)$. Then if $k \in I(l)$ and $k \beta=p \alpha+h$ as in Lemma 7, we have

$(k-\mu) \beta=(p-c) \alpha+h-e \cdot(l-1)$, where $0 \leq-e \cdot[h-e \cdot(l-1)] \leq \alpha-1$.

When $e=+1$ this leads to $\varepsilon(k-\mu)=(-1)^{p-c-1}$, and when $e=-1$ it leads to $\varepsilon(k-\mu)=(-1)^{p-c}$. In both cases, $\varepsilon(k-\mu)=e \cdot(-1)^{p-c-1}$, but because 
Lemma 7 asserts $\delta(k)=e \cdot(-1)^{p-1}$ we get (5.3). When $\alpha$ is odd the pair $(\mu-\alpha, c-\beta)$ can replace $(\mu, \alpha)$ with change of parity of $\mu$, and when $\alpha$ is even the replacement changes the sign of $\mu$. This completes the proof of Lemma 8.

\section{HECKOID GROUPS ARE HOMOMORPHIC IMAGES OF 2-BRIDGE KNOT OR LINK GROUPS}

We use the hypotheses and notation of $\S 5$ throughout this section.

Theorem 3. Given $K=(\alpha, \beta)$ and $K^{*}(e)=\left(\alpha^{r} q, \alpha^{r-1} q(\alpha-\beta)+e\right)$, where $e= \pm 1, r \geq 2$, and $q=2 n+1$ or $2 n+2$ with $n \geq 1$, write $\tilde{u}=\left(t-t^{-1}\right)^{2}-u$ when $\alpha$ is odd, and $\tilde{u}=\left(s-s^{-1}\right)\left(t-t^{-1}\right)-u=-\zeta(t, s, u)$ when $\alpha$ is even.
A. When $\alpha$ and $q$ are odd, $f_{n}\left(v_{K}(t, \tilde{u})\right) \mid \Phi_{K^{*}(e)}(t, u)$.
B. When $\alpha$ is even, $q$ odd, $f_{n}\left(v_{L}(t, s, \tilde{u})\right) \mid \Phi_{L^{*}(e)}(t, s, u)$.
C. When $\alpha$ is odd, $q$ even, $\quad g_{n}\left(v_{K}(t, \tilde{u})\right) \mid \Phi_{L^{*}(e)}(t, t, u)$.
D. When $\alpha$ and $q$ are even, $g_{n}\left(v_{L}(t, s, \tilde{u})\right) \mid \Phi_{L^{*}(e)}(t, s, u)$.

Proof. The sequence $\vec{\delta}$ is the exponent sequence of a word $\hat{w}=\tilde{w}^{*} x_{1}^{\delta\left(\alpha^{*}\right)}$ of $\pi \widetilde{K}_{e}^{*}$. With $C=C\{t\}, D$ either $D\{t, u\}$ or $D\{s, u\}$, as appropriate for the parity of $\alpha$, we set $\widehat{W}=\hat{w}(C, D)=\cdots C^{\delta(k)} D^{\delta(k+1)} \cdots C^{\delta\left(\alpha^{*}\right)}$. For $l=1, \ldots, \alpha$ let $\Delta(l)$ be the sequence $(\delta(\nu), \delta(\nu+1), \ldots, \delta(l A))$, where $\nu=(l-1) A+1$. We write $\widehat{W}=Z_{1} \cdots Z_{\alpha}$, where the subword $Z_{l}$ has exponent sequence $\Delta(l)$.

Lemma 9. For $l=1, \ldots, \alpha$ :

( $q$ is odd): In $\operatorname{PSL}_{2}\left(R /\left(f_{n}(v)\right)\right)$, for odd $\alpha, Z_{l}=U_{e, n}$. For even $\alpha, Z_{l}=E$ if $r>2$. If $r=2$ then $Z_{l}=U_{e, n}$ for odd $l$, and $Z_{l}=J\left(U_{e, n}\right)$ of Lemma 6 for even $l$.

( $q$ is even): $Z_{l}=E$ in $\operatorname{PSL}_{2}\left(R /\left(g_{n}(v)\right)\right)$.

Proof. Throughout the argument all matrices are taken to be in $\mathrm{PSL}_{2}$ of the quotient ring of $R$ appropriate for the parity of $q$. Consider first the case $l=1$ :

$$
Z_{1}=C^{\delta(1)} D^{\delta(2)} \ldots C^{\delta(A)} \quad(\alpha \text { odd }), \quad Z_{1}=D^{\delta(1)} C^{\delta(2)} \ldots C^{\delta(A)} \quad(\alpha \text { even }) .
$$

By Lemma $\delta$ the exponent sequence of $Z_{1}$ is $\left(\varepsilon_{1}, \cdots, \varepsilon_{A}\right)$, and it is easy to see that this is the exponent sequence of $U_{e, n}^{p}$ when $q$ is odd, and of $V_{e}^{(n+1) p}$ when $q$ is even, where $p=\alpha^{r-2}$. It follows that $Z_{1}=U_{e, n}^{p}$ for odd $q$, and $Z_{1}=V_{e}^{(n+1) p}=E$ for even $q$. Also $p$ is odd if $\alpha$ is odd or $r=2$, and then $Z_{1}=U_{e, n}$. For even $\alpha$ and $p, Z_{1}=E$. We finish the proof by arguing that the other $Z_{l}$ are conjugate to $Z_{1}$ or $\bar{Z}_{1}$, the word obtained from $\left(-\varepsilon_{1}, \ldots,-\varepsilon_{A}\right)$, or $J\left(Z_{1}\right)$ or $J\left(\bar{Z}_{1}\right)$.

When $l>1$ Lemma 8 asserts that

$$
\Delta(l)=(-1)^{c}(\varepsilon(\nu-\mu), \varepsilon(\nu+1-\mu), \ldots, \varepsilon(l A-\mu)) .
$$

If $\mu>0$ then another expression for $Z_{l}$ has exponent sequence $(-1)^{c}$ times

$$
\begin{aligned}
& \varepsilon(\nu-\mu), \varepsilon(\nu+1-\mu), \ldots, \varepsilon(\nu-1), \\
& \varepsilon(\nu), \varepsilon(\nu+1), \ldots, \varepsilon(l A), \\
& -\varepsilon(l A),-\varepsilon(l A-1), \ldots,-\varepsilon(l A+1-\mu) .
\end{aligned}
$$


The letter under $\varepsilon(\nu-\mu)$ is the initial letter of $Z_{l}$, and the letters under the exponents alternate between $C$ and $D$ from the beginning of the top line through the end of the second, and on the third. The letters under $\varepsilon(l A)$ and $-\varepsilon(l A)$ are the same, so that the third line cancels the part of the second beyond $l A-\mu$. We now have to distinguish four cases, as in Theorem 3. For case A we have $\alpha$ and $q$ are odd, whence $A=\alpha^{r-1} q$ is odd and the letters under $\varepsilon(\nu-1),-\varepsilon(l A)$ of $(6.1)$ are different. The several expressions for $U_{e, n}$ in the Corollary to Theorem 1 tell us that the subproduct $Z$ corresponding to the middle line of (6.1) is $U_{e, n}^{p}=U_{e, n}$. The relations of this Corollary allow us to push $U_{e, n}$ to the right end of the product, so that, in bastard notation,

$$
\begin{aligned}
Z_{l}= & \varepsilon(\nu-\mu), \varepsilon(\nu+1-\mu), \ldots, \varepsilon(\nu-1), \\
& \varepsilon(l A), \varepsilon(l A-1), \ldots, \varepsilon(l A+1-\mu) \cdot U_{e, n} .
\end{aligned}
$$

Now the letters under $\varepsilon(\nu-h), \varepsilon(l A+1-h)$ are the same for $h=1, \ldots, \mu$. The skew periodicity $\varepsilon(k+\alpha)=-\varepsilon(k)$ leads to $\varepsilon(\nu-h)=-\varepsilon(l A+1-h)$, whence the product in front of $U_{e, n}$ collapses to $E$, leaving $Z_{l}=U_{e, n}=Z_{1}$ when $\alpha$ and $q$ are odd, $\mu>0$. But the parity of $\mu$ is irrelevant here, so we always choose $\mu>0$ except when $\alpha$ is odd and $q$ is even.

When $\alpha$ is even but $q$ is odd, $A$ is even and the letters under $\varepsilon(\nu-1)$, $-\varepsilon(l A)$ of $(6.1)$ are equal. If the letter under $\varepsilon(\nu)$ is $D$ then the product $Z$ for the middle line of (6.1) is $U_{e, n}$, and one sees that the cancellation argument for $\alpha q$ odd now applies, to give $Z_{l}=Z_{1}$. If the letter under $\varepsilon(\nu)$ is $C$ then $Z$ is $J\left(U_{e, n}\right)$ of Lemma 6 , and the argument gives $Z_{l}=J\left(Z_{1}\right)$ instead. If $Z_{1}=E$ then $Z_{l}=J\left(Z_{l}\right)=E$. If $Z_{1} \neq E$ we still have to show that the parity of $l$ determines whether $Z=Z_{1}$ or not. For even $\alpha, \nu$ is odd and the letter under $\varepsilon(\nu-\mu)$ is always $D$, whence the letter for $\varepsilon(\nu)$ is $D$ when $\mu$ is even, and is $C$ when $\mu$ is odd. The defining congruence for $\mu$ is $\mu \beta \equiv e \cdot(l-1)$ $(\bmod \alpha)$, implying $\mu$ and $l$ have opposite parity. Therefore $Z_{l}=Z_{1}$ for odd $l$, and $Z_{l}=J\left(Z_{1}\right)$ for even $l$, when $\alpha$ is even, $q$ is odd, and $r=2$.

In the cases where $q$ is even, $A=\alpha^{r-1} q$ is even and the letters under $\varepsilon(\nu-1),-\varepsilon(l A)$ are the same. Also $\varepsilon(k+A)=\varepsilon(k)$, so $\varepsilon(\nu-h)=\varepsilon(l A+1-h)$ for $h=1, \ldots, \mu$. Hence $Z_{l}$ will be $E$ if we can show that the product $Z$ for the middle line of $(6.1)$ is $E$. For even $q, Z$ is one of $V_{e}^{(n+1) p}, \bar{V}_{e}^{(n+1) p}$, or $J$ of these, and by Lemma 6 and the Corollary to Theorem 2 we get $Z=E$. For odd $q$, if the letter under $\varepsilon(\nu)$ is $C$ then $Z$ is $V_{e}^{(n+1) p}$ or $\bar{V}_{e}^{(n+1) p}$, whence $Z=E$. The remaining case is $\alpha$ odd, $q$ even, and the letter under $\varepsilon(\nu)$ is $D$. Here we have to replace $\mu$ by $\mu-\alpha<0$ to change the parity. We write $Z_{l}$ as a new product, with exponent sequence $(-1)^{c}$ times

$$
\begin{aligned}
& -\varepsilon(\nu-\mu-1),-\varepsilon(\nu-\mu-2), \ldots,-\varepsilon(\nu), \\
& \varepsilon(\nu), \varepsilon(\nu+1), \ldots, \varepsilon(l A), \\
& \varepsilon(l A+1), \ldots, \varepsilon(l A-\mu) .
\end{aligned}
$$

The middle line product $Z$ for (6.2) now begins with $C$, so $Z=E$ as before, and then the rest of the new product cancels to $E$. This completes the proof of Lemma 9.

To complete the proof of Theorem 3 we continue the convention that all matrices are considered to be in $\mathrm{PSL}_{2}$ of the appropriate quotient ring of $R$. 
Then $\widehat{W}=E$ when $q$ is even or if $\alpha$ is even and $r>2$. If $\alpha$ and $q$ are even and $r=2$ then $\widehat{W}=(U J)^{\alpha / 2}$, where $U=U_{e, n}$ and $J=J(U)$. The Corollary to Theorem 2 and Lemma 6 imply $U J=J U$, hence $\bar{W}=U^{\alpha / 2} J^{\alpha / 2}$, which is $E$ if $4 \mid \alpha$, and $U J$ otherwise. Now $U J C=U C^{-1} J=C U J$, so in all cases where $\alpha q$ is even, $\widehat{W} C=C \widehat{W}$. This gives $\widetilde{W} C=C \widetilde{W}$, where $\widetilde{W}=\widehat{W} C^{-\delta\left(\alpha^{*}\right)}=\widetilde{w}^{*}(C, D)$, which is equivalent to $\Phi_{\widetilde{L}^{*}(e)}=0$. This conclusion expands to the following three assertions:

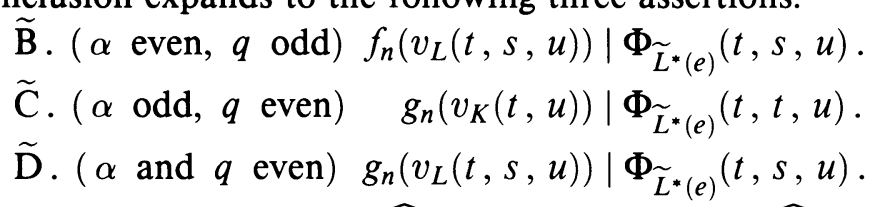

When $\alpha q$ is odd then $\widehat{W}=U_{e, n}^{\alpha}=U_{e, n}$ and $\widehat{W} C=D^{-1} \widehat{W}$ by Theorem 1. Therefore $\widetilde{W}^{*} C=D^{-1} \widetilde{W}^{*}$, where $\widetilde{W}^{*}=\widehat{W} C^{-\delta\left(\alpha^{*}\right)}=\tilde{w}^{*}(C, D)$. This is equivalent to

$\widetilde{\mathrm{A}}$. $(\alpha$ and $q$ odd $) f_{n}\left(v_{K}(t, u)\right) \mid \Phi_{\widetilde{K}^{*}(e)}(t, u)$.

The final step is the conversion of $\widetilde{\mathrm{A}}-\widetilde{\mathrm{D}}$ to the versions $\widetilde{\mathrm{A}}-\widetilde{\mathrm{D}}$ in Theorem 3. The matrix $P_{B}$ of part B of Lemma 5 exists when $f_{n}(v)=0$ or $g_{n}(v)=0$, and $P_{B} \widetilde{W}^{*}(t, u) P_{B}^{-1}=W^{*}(t, \tilde{u}), \tilde{u}=\left(t^{-1}-t\right)^{2}-u$, when $\alpha$ and $q$ are odd. Hence $P_{B}$ transforms $\widetilde{W}^{*} C\{t\}=D^{-1}\{t, u\} \widetilde{W}^{*}$ to $W^{*} C\{t\}=D\{t, \tilde{u}\} W^{*}$, so $\Phi_{K^{*}(e)}(t, \tilde{u})=\Phi_{\widetilde{K}^{*}(e)}(t, u)$. Because $\tilde{\tilde{u}}=u$, replacing $u$ by $\tilde{u}$ in $\widetilde{\mathrm{A}}$ now gives assertion $\mathrm{A}$ of Theorem 3. This argument works with obvious modifications to prove the rest of Theorem 3 .

\section{FACTORIZATION OF THE PRECUSP POLYNOMIAL FOR A 2-BRIDGE KNOT}

Let $K=(\alpha, \beta)$ be a 2-bridge normal form, excluding $O=(1,1)$, where Theorem 4 would degenerate. Then $\Phi_{K}(t, u)$ and $v_{K}(t, u)=u \Phi_{K}^{2}$ are available, and reduce in the special case of parabolic representations ( $p$-reps) to $\Lambda_{K}(u)=\Phi_{K}(1, u)$ and $v_{K}(u)=u \Lambda_{K}^{2}$. Using the normal form $A=C\{1\}$ and $B_{u}=B\{u\}=D\{1, u\}$ of $(0.1)$, we set $W=W(1, u)=w_{K}\left(A, B_{u}\right)$ and $V_{e}=W A^{e} W^{-1} B^{-e}$, where $e= \pm 1$. The word $V_{e}(u)$ has trace $2+v_{K}(u)$ and becomes parabolic, of trace -2 , when $4+v_{K}(u)=0$. We call $4+v_{K}(u)$ the precusp polynomial of $K$. It is a monic polynomial of degree $\alpha$ in $\mathbb{Z}[u]$, and, like $\Phi_{K}(t, u)$ and $\Lambda_{K}(u)$, depends on the specific normal form $K$ rather than the knot type of $K$.

Theorem 4. Corresponding to the 2-bridge knot normal form $K=(\alpha, \beta)$ there is a kmot group $\pi K^{*}$ of determinant $\alpha$ with nonabelian representation polynomial $\Phi_{\pi K^{*}}(t, u)$ such that $\Phi_{\pi K^{*}}(1,-u)$ divides the precusp polynomial of $K$.

We abbreviate $\Phi_{\pi K^{*}}(1, u)$ to $\Lambda^{*}(u)$. The shape of $\Lambda^{*}(-u)$ claimed in Theorem $\mathrm{C}$ of the introduction is simply a consequence of $\operatorname{det}\left(\pi K^{*}\right)=\alpha$. There is no direct analogue of Theorem 4 for 2-bridge kmot groups or for 2-bridge link groups. For if $\pi K$ is the proper kmot group of determinant 7 mentioned in $\S 1$, then the "precusp polynomial" $4+v_{\pi K}(u)$ for it is irreducible, while for several other proper kmot groups, $4+v$ has proper factors but none of the shape of Theorem C. Experience suggests that the cusp polynomial $4+v_{L}(1,1, u)$ for a 2-bridge link is always irreducible. Finally, Theorem 4 definitely requires that 
$t=1$ in $4+v_{K}$, because for the trefoil knot $(3,1), 4+v_{K}(t, u)$ is irreducible in $\overline{\mathbb{Q}}\left[t, t^{-1}, u\right]$, where $\overline{\mathbb{Q}}$ is an algebraic closure of $\mathbb{Q}$.

Proof of Theorem 4 . We begin by finding a suitable element of the image $\pi K \theta$ of a 2-bridge kmot group of determinant $\alpha$ whose trace is -2 when $x_{1} \theta=A$ and $x_{2} \theta=B\{\omega\}$, using the standard presentation (1.1). Although the relation $\bar{w} x_{1}=x_{2} \bar{w}, \bar{w}$ defined by (1.3), does not necessarily hold in $\pi K$, its image $\bar{W} A=B \bar{W}, \bar{W}=\bar{w}(A, B)=w\left(A^{-1}, B^{-1}\right)$, does hold in $\pi K \theta$. For if $R=P_{C}$ of Lemma 5 , then

$$
R=\left[\begin{array}{cc}
i & 0 \\
0 & -i
\end{array}\right], \quad R A R^{-1}=A^{-1}, \quad R B\{u\} R^{-1}=B\{u\}^{-1},
$$

so conjugation by $R$ converts $W A=B W$ to $\bar{W} A^{-1}=B^{-1} \bar{W}$, which is equivalent to $\bar{W} A=B \bar{W}$. Since $\theta$ is a $p$-rep, $\Phi_{\pi K}(1, \omega)=w_{11}(1, \omega)+0 \cdot w_{12}=0$, and

$$
\begin{gathered}
W=\left[\begin{array}{cc}
0 & w_{12} \\
-\omega w_{12} & w_{22}
\end{array}\right], \quad \bar{W}=\left[\begin{array}{cc}
0 & -w_{12} \\
\omega w_{12} & w_{22}
\end{array}\right], \\
\bar{W}^{-1} W=\left[\begin{array}{cc}
-1 & 2 w_{12} w_{22} \\
0 & -1
\end{array}\right],
\end{gathered}
$$

as in $[4, \mathrm{I}, \S 3]$. So $G=\bar{W}^{-1} W$ commutes with $A$ and has trace -2 . The formal expression of $G$ as a word on $A, B$ is

$$
G=B^{\varepsilon_{\alpha-1}} A^{\varepsilon_{\alpha-2}} B^{\varepsilon_{\alpha-3}} \cdots B^{\varepsilon_{2}} A^{2 \varepsilon_{1}} B^{\varepsilon_{2}} A^{\varepsilon_{3}} \cdots B^{\varepsilon_{\alpha-1}},
$$

which is not suitable for later use because of the even exponent $2 \varepsilon_{1}$. However, for $e= \pm 1$, the word

$$
G_{e}:=\bar{W}^{-1} B^{e} W A^{e}=B^{\varepsilon_{\alpha-1}} A^{\varepsilon_{\alpha-2}} \cdots A^{\varepsilon_{1}} B^{e} A^{\varepsilon_{1}} \cdots B^{\varepsilon_{\alpha-1}} A^{e}
$$

has all the right properties: $\operatorname{tr}\left(G_{e}\right)=-2$, length $\left(G_{e}\right)=2 \alpha$, the exponent sequence of $G_{e}$ agrees with that of $w_{\pi K}$ in the first $\alpha-1$ places, all exponents are \pm 1 , and $G_{e}$ commutes with $A$ in $\pi K \theta$ because $G_{e}=G A^{2 e}$.

Given a 2-bridge knot normal form $K=(\alpha, \beta)$ we have the associated pair $\widetilde{K}=(\alpha, \tilde{\beta}), \tilde{\beta}=\alpha-\beta$, of $\S 5$, and the standard presentations (1.1) of $\pi K$ and $(5.1)$ of $\pi \widetilde{K}$. Set $B=B\{u\}, W=w(A, B)$, and $\widetilde{W}=\tilde{w}(A, B)=$ $w\left(A, B^{-1}\right)$, and for $e= \pm 1$ set $V_{e}=V_{e}(u)=W A^{e} W^{-1} B^{-e}$. Since $B^{-1}=$ $B\{-u\}, \quad \widetilde{V}_{e}=\widetilde{V}_{e}(u):=\widetilde{W} A^{e} \widetilde{W}^{-1} B^{e}=V_{e}(-u)$. We extend the exponent sequence $\vec{\varepsilon}$ of $w$ to length $2 \alpha$ by (5.2), and then $\vec{\varepsilon}$ is the exponent sequence of $V_{e}$. The exponent sequence $\vec{\delta}$ of $\widetilde{V}_{e}$ also has length $2 \alpha$ and $\delta(k)=(-1)^{k+1} \varepsilon(k)$, so that $\delta(k+\alpha)=\delta(k)$ for $1 \leq k \leq \alpha$. We will take the subscripts of $\vec{\delta}$ modulo $2 \alpha$, i.e., $\delta(k+2 \alpha) \equiv \delta(k)$.

There exists a unique even integer $h$, where $1<h<\alpha$, such that $h \tilde{\beta}=$ $q \alpha+(\alpha+f) / 2$ with integral $q$, and $f \in\{1,-1\}$. At this point we choose $e=\delta(2 h)$, and drop the subscript, so that $\widetilde{V}(u)$ is now completely determined by $K$. Circular permutation of $\vec{\delta}$ corresponds to conjugation of $\widetilde{V}$ by a specific word $P$ on $A, B$, and we consider

$P \widetilde{V} P^{-1}$ with exponent sequence $\delta(h+1), \delta(h+2), \ldots, \delta(h+2 \alpha)$. 
We claim: for $1 \leq k<\alpha, \delta(h+k)=\delta(h-k)$. To see this, say $k \tilde{\beta}=p \alpha+t$, where $p, t \in \mathbb{Z}, 1 \leq t<\alpha$, then

$$
(h+k) \tilde{\beta}=(q+p) \alpha+\frac{a+f}{2}+t, \quad(h-k) \tilde{\beta}=(q-p) \alpha+\frac{\alpha+f}{2}-t .
$$

When $t \neq(\alpha \pm 1) / 2$ it is easy to derive $\delta(h+k)=\delta(h-k)$ from this and (5.2). For $k=h$ we have $\delta(2 h)=e=\delta(\alpha)=\delta(0)$, and likewise for $k=\alpha-h$. This proves the claim.

Let $\vec{\gamma}=\left(\gamma_{1}, \ldots, \gamma_{\alpha-1}\right)$, where $\gamma(k):=\delta(h+k)$. The claim implies that $\gamma(\alpha-k)=\gamma(k)$, so that $\vec{\gamma}$ is the exponent sequence of the defining word $w^{*}=$ $x_{1}^{\gamma_{1}} x_{2}^{\gamma_{2}} \cdots x_{2}^{\gamma_{\alpha-1}}$ of a kmot group $\pi K^{*}$ of determinant $\alpha$. Hence the exponent sequence of $P \tilde{V} P^{-1}$ is the same as that of $\left(\bar{w}^{*}\right)^{-1} x_{2}^{\delta(h)} w^{*} x_{1}^{\delta(h)}$, i.e., $P \widetilde{V} P^{-1}$ and $G_{\delta(h)}$ for $\pi K^{*}$ have the same exponent sequence, and the initial letter of both words is $B$ (remember $h$ is even). Therefore if $\omega$ is any root of $\Lambda^{*}(u)=0$ and $\theta=\theta\{\omega\}$ is the corresponding $p$-rep of $\pi K^{*}$, then $P \widetilde{V} P^{-1}\{\omega\}=G_{\delta(h)} \in$ $\pi K^{*} \theta$. This means that $\Lambda^{*}(\omega)=0$ implies that $\operatorname{tr}(\tilde{V})=2+v_{K}(-\omega)=-2$, and $4+v_{K}(-\omega)=0$. Because $\Lambda^{*}(u)$ has no repeated factors (Lemma 3 of [7]) we get $\Lambda^{*}(u) \mid 4+v_{K}(-u)$. Replace $u$ by $-u$ here to complete the proof of Theorem 4 .

A few special cases of Theorem 4 may be of interest. Perhaps they could be proved by direct inductions, as a rather challenging project in recreational mathematics.

A. $K=(\alpha, 1)$. Then $K^{*}=(\alpha, \alpha-2)$, a twist knot. Here Theorem 4 can be expressed using Morgan-Voyce polynomials and there are two subcases.

Aa. $\alpha=4 n+1$, then $1+u f_{n}(u) g_{n-1}(u) \mid 4-u f_{2 n}^{2}(u)$.

Ab. $\alpha=4 n+3$, then $1-u f_{n}(u) g_{n}(u) \mid 4-u f_{2 n+1}^{2}(u)$.

B. $K=(\alpha,(\alpha \pm 1) / 2)$, a twist knot. Then $K^{*}=K$.

Ba. $K=(4 n+1,2 n+1)$, then $f_{n}\left(u^{2}\right)-u g_{n-1}\left(u^{2}\right) \mid 4+u\left[f_{n}\left(u^{2}\right)+u g_{n-1}\left(u^{2}\right)\right]^{2}$.

Bb. $K=(4 n+3,2 n+1)$, then $f_{n}\left(u^{2}\right)+u g_{n}\left(u^{2}\right) \mid 4+u\left[f_{n}\left(u^{2}\right)-u g_{n}\left(u^{2}\right)\right]^{2}$.

For the twist knot $(7,5)$ the kmot group $\pi K^{*}$ of Theorem 4 is the proper kmot group mentioned in $\S 1$. The factor $\Lambda^{*}(-u)$ or its cofactor in the precusp polynomial may itself be reducible, and calculations using MAPLE suggest that reducibility is both frequent and eclectic. We predict that the precusp polynomial will always have an irreducible factor $\operatorname{cusp}_{K}(u) \in \mathbb{Z}[u]$ which has constant term 4 and a root $\omega$ on bdry $(\mathscr{R})$, as described in the introduction. This will be the cusp polynomial for the normal form $K$. We predict also that $\operatorname{deg}\left(\operatorname{cusp}_{K}(u)\right)$ depends partly on the size of the symmetry group for Thurston's link $L$ associated to the cusp for $K$.

\section{HECKOID ALGEBRA FOR MORE THAN TWO GENERATORS}

At this writing this topic consists of special cases of one incompletely worked class of examples, the generalized pretzel knots $K(n, r)$, abbreviating a row $(n, n, \ldots, n)$ of length $r \geq 3$. We have only considered the case $r n$ is odd, and our attempt to get an analogue of Theorem 1 guided by calculations with MAPLE bogged down in complexity for $n>1$, where the matter currently rests. It must be possible to organize these huge expressions into blocks of manageable subexpressions in a meaningful and useful way, but this will take 
time and MAPLE is not good for this game. For $n=1, K(n, r)$ is actually a 2-bridge torus knot, but we are not using the 2-bridge formalism. We did manage to get the analogue of Theorem 1 for the Heckoid groups for $K(1, r)$. If we give up on the representation curves for the Heckoid groups for $K(n, r)$ and simply go for their $p$-reps the algebra simplifies so much that we can state complete results using Morgan-Voyce polynomials. Therefore we can find the actual Heckoid groups for the knots $K(n, r)$, e.g., the modest example of [6, 1], and we expect to get the "cusp groups," the algebraic limit of the $p$-rep for the $q$ th Heckoid group of $K(n, r)$ as $q \rightarrow \infty$, without much trouble, although we have not tried yet.

The real challenge in this subject is not the algebra but the geometry. If a noncompact 3-manifold $M$ admits a complete hyperbolic structure (of finite volume ?) and also an axis of rotational symmetry, does $M$ also admit complete hyperbolic orbifold structures of infinite volume corresponding to Kleinian groups of second kind? If so this would at least explain the even Heckoid groups. The most general context for Theorems 3 and 4 is unguessable at present.

\section{REFERENCES}

1. M. Grayson, The orbit space of a Kleinian group: Riley's modest example, Math. of Comp. $\mathbf{4 0}$ (1983), 633-646.

2. T. Jorgensen, Compact 3-manifolds of constant negative curvature fibering over the circle, Ann. of Math. (2) 106 (1977), 61-72.

3. T. Ohtsuki, Ideal points and incompressible surfaces in two-bridge knot complements, preprint, 1990.

4. R. Riley, Parabolic representations of knot groups. I, II, Proc. London Math. Soc. (3) 24 (1972), 217-242; 31 (1975), 495-512.

5. __ Knots with the parabolic Property P, Quart. J. Math. Oxford (2) 25 (1974), 273-283.

6. A Applications of a computer implementation of Poincaré's Theorem on fundamental polyhedra, Math. Comp. 40 (1983), 607-632.

7. $\ldots$,Nonabelian representations of 2-bridge knot groups, Quart. J. Oxford (2) 35 (1984), 191208.

8. H. Schubert, Knoten mit zwei Brücken, Math. Z. 65 (1956), 133-170.

9. W. Thurston, The geometry and topology of 3-manifolds (to appear).

Department of Mathematical Sciences, State University of New York at BinghamTON, BINGHAMTON, NEW YORK 13901

E-mail address: bob@nijlpaard.math.binghamton.edu 